\title{
Well-posedness of a higher-order Schrödinger-Poisson-Slater system
}

\section{Saber Trabelsi ${ }^{*}$}

\section{${ }^{*}$ Correspondence:}

saber.trabelsi@kfupm.edu.sa

'Department of Mathematics \& Statistics, King Fahd University of Petroleum and Minerals, Dhahran, Kingdom of Saudi Arabia

\begin{abstract}
In this paper, we show the global well-posedness of a higher-order nonlinear Schrödinger equation. Specifically, we consider a system of infinitely many coupled higher-order Schrödinger-Poisson-Slater equations with a self-consistent Coulomb potential. We prove the existence and uniqueness global in time of solutions in $L^{2}\left(\mathbb{R}^{3}\right)$ and in the energy space.
\end{abstract}

MSC: 35Q55; 35Q41

Keywords: Nonlinear Schrödinger equations; Nonlinear Schrödinger-Poisson equations

\section{Introduction}

This paper is devoted to the mathematical analysis of the following higher-order Schrödinger-Poisson-Slater mixed system (SPS in short) in three space dimensions:

$$
i \hbar \frac{\partial}{\partial t} \psi_{k}=-\sum_{j=0}^{J} \frac{\alpha(j) \hbar^{2 j}}{m^{2 j-1} c^{2 j-2}} \Delta^{j} \psi_{k}+c_{c} \frac{1}{|x|} \psi_{k}+c_{p}\left(\rho_{\psi} \star \frac{1}{|x|}\right) \psi_{k}-c_{s} \rho_{\psi}^{\frac{1}{3}} \psi_{k} .
$$

The wavefunction $\psi_{k}$ depends on time $t$ and space $x \in \mathbb{R}^{3}$. The constants $m, c$ and $\hbar$ denote the mass of a particle, the speed of light, and the reduced Planck constant, respectively. The constants $c_{c}, c_{p}$ and $c_{s}$ denote physical interaction constants; the subscripts ${ }_{c},{ }^{\cdot}{ }_{p}$ and ${ }_{s}$ stand for Coulomb, Poisson and Slater, respectively. The SPS system can be either repulsive $\left(c_{p}>0\right)$ or attractive $\left(c_{p}<0\right)$. The differential operator $\Delta$ is the Laplace operator, and $\star$ denotes the convolution operator in $\mathbb{R}^{3} . \rho_{\psi}$ denotes the charge density and is given by $\rho_{\psi}:=\sum_{j \in \mathbb{N}} \gamma_{i}\left|\psi_{j}\right|^{2}$, where $\left\{\gamma_{j}, j \in \mathbb{N}\right\} \subset \ell^{1}(\mathbb{N})$ is the set of ocupation numbers and are such that $\gamma_{j} \geq 0$ and $\sum_{j \in \mathbb{N}} \gamma_{j}=1$. The nonlinear term $-c_{s} \rho_{\psi}^{\frac{1}{3}} \psi_{k}$ was introduced by Slater as a local correction to the exchange term in the Hartree-Fock equations. This correction should be understood, following Dirac and Slater, as a quantum effect in contrast with the Poisson term which has a classical counterpart, we refer to $[1,2]$ for more details, and to [3-5] for an heuristic justification of this term. The difference between system (1) and the classical SPS system is that the kinetic operator $\frac{\hbar}{2 m} \Delta$ is replaced by the higher-order operator $\sum_{j=0}^{J} \frac{\alpha(j) \hbar^{2 j}}{m^{2 j-1} c^{2 j-2}} \Delta^{j}$ where $J \in \mathbb{N}^{\star}$. This idea was developed in [6, 7] and is based on Einstein's mass-energy equivalence and a finite expansion approach. This model is adapted for particles with velocity less than $\frac{c}{\sqrt{2}}$.

(c) The Author(s) 2018. This article is distributed under the terms of the Creative Commons Attribution 4.0 International License (http://creativecommons.org/licenses/by/4.0/), which permits unrestricted use, distribution, and reproduction in any medium, provided you give appropriate credit to the original author(s) and the source, provide a link to the Creative Commons license, and indicate if changes were made. 
System (1) belongs to the family of higher-order Schrödinger equations that have been developed in, e.g., $[7,8]$. These are Schrödinger-type equations involving higher-order Schrödinger operator converging, in principle, to the semi-relativistic bound-state equation called the spinless Salpeter equation; see, e.g., [9-12]. The semi-relativistic spinless Salpeter equation is of high importance in particle physics and provides a particularly well defined approximation to the Bethe-Salpeter formalism designed for the Lorentzcovariant description of bound states within relativistic quantum field theory. The spinless Salpeter equation can be formally obtained using a three-dimensional reduction and suitable justified physical hypotheses. More importantly, it can be regarded as a generalization of the classical Schrödinger equation with the inclusion of the relativistically correct freeparticle kinetic energy. We refer the reader to [6] for a more detailed discussion of the physical background and application of the higher-order Schrödinger equations (also see [7] for an application to the case of alpha particles and see [13,14] for alternative applications).

In the sequel, we assume that the sequence of nonnegative real numbers $\left\{\gamma_{j}, j \in \mathbb{N}\right\} \subset$ $\ell^{1}(\mathbb{N})$ are fixed. That is, they do not depend on time as in the time dependent multiconfiguration models (see e.g. [15] ). Next, we recall the definition of some functional spaces, originally introduced in $[16,17]$. We denote $\gamma:=\left(\gamma_{1}, \gamma_{2}, \ldots\right)$ and we define, for all $1 \leq p \leq q \leq+\infty$, the $L^{p}(\gamma)$ space as follows:

$$
L^{p}(\gamma):=\left\{\psi=\left(\psi_{i}\right)_{i \in \mathbb{N}},\|\psi\|_{L^{p}(\gamma)}=\left(\sum_{j \in \mathbb{N}} \gamma_{j} \int_{\mathbb{R}^{3}}\left|\psi_{j}\right|^{p} d x\right)^{1 / p}<+\infty\right\} .
$$

For a given time interval $I$, we denote $L_{I}^{q, p}(\gamma):=L^{q}\left(I ; L^{p}(\gamma)\right)$. We shall use the notation $L_{T}^{q, p}(\gamma)$ when $I=[0, T]$. Also, we shall use the shorthand notation $L_{\mathrm{loc}}^{q, p}(\gamma)$ and $L^{q, p}(\gamma)$ for $L_{\mathrm{loc}}^{q}\left([0,+\infty) ; L^{p}(\gamma)\right)$ and $L^{q}\left([0,+\infty) ; L^{p}(\gamma)\right)$, respectively. Now, we introduce

$$
\mathfrak{B}_{I}^{q, p}(\gamma):=L_{I}^{\infty, 2}(\gamma) \cap L_{I}^{q, p}(\gamma), \quad \mathfrak{B}_{I}^{\prime q, p}(\gamma):=L_{I}^{1,2}(\gamma) \cap L_{I}^{\frac{q}{q-1}, \frac{p}{p-1}}(\gamma)
$$

equipped with the norms

$$
\begin{aligned}
& \|\psi\|_{\mathfrak{B}_{I}^{q, p}(\gamma)}=\|\psi\|_{L_{I}^{\infty, 2}(\gamma)}+\|\psi\|_{L_{I}^{q, p}(\gamma)}, \\
& \|\psi\|_{\mathfrak{B}_{I}^{q, p}(\gamma)}=\inf _{\psi=\psi^{1}+\psi^{2}}\left(\left\|\psi^{1}\right\|_{L_{I}^{1,2}(\gamma)}+\left\|\psi^{2}\right\|_{L_{I}^{\frac{q}{q-1}, \frac{p}{p-1}(\gamma)}}\right) .
\end{aligned}
$$

Eventually, we define the set

$$
\mathfrak{J}:=\left\{(q, p) \text { such that } 2 \leq p \leq+\infty, J \in \mathbb{N}^{\star} \backslash\{1\} \text { and } \frac{2}{q}=\frac{3}{J}\left(\frac{1}{2}-\frac{1}{p}\right)\right\} .
$$

The main result of this paper is the following.

Theorem 1.1 Let $J \geq 2$ and $\phi=\left(\phi_{i}\right)_{i \in \mathbb{N}}$ be a set of initial data in $L^{2}(\gamma)$ and $(q, p) \in \mathfrak{J}$ such that $3<p \leq 6$. Then the SPS system (1) has a unique solution $\psi(t, x)$ satisfying

$$
\psi(t, x) \in C^{0}\left([0,+\infty), L^{2}(\gamma)\right) \cap L^{q}\left([0,+\infty), L^{p}(\gamma)\right) .
$$


In addition, if $\phi=\left(\phi_{i}\right)_{i \in \mathbb{N}} \in H^{2 J}(\gamma)$, then

$$
\psi(t, x) \in C^{0}\left([0,+\infty), H^{2 J}(\gamma)\right) \cap C^{1}\left([0,+\infty), L^{2}(\gamma)\right) .
$$

This theorem shows the well-posedness of system (1) with initial data in $L^{2}$ instead of the energy space. Also, it is worth mentioning that Theorem 1.1 can be extended as follows.

Corollary 1.2 Let $\phi=\left(\phi_{i}\right)_{i \in \mathbb{N}}$ be a set of initial data in $H^{J}(\gamma)$, then the SPS system (1) has a unique solution $\psi(t, x)$ satisfying $\psi(t, x) \in C^{0}\left([0,+\infty), H^{J}(\gamma)\right)$.

There is a very rich mathematical literature dedicated to the analysis of the Hartree and Hartree-Fock equations. The well-posedness in the energy space $H^{1}\left(\mathbb{R}^{3}\right)$ of the HartreeFock equations $(\delta=1, k=1, \ldots, N)$ was obtained in [18] (see also [19]). An $L^{2}$ theory was established, independently, in [16] and [17]. The authors established Strichartz' type estimates for the free Schrödinger semigroup in some weighted spaces, and used a contraction argument to conclude. In [20], the authors showed the well-posedness of the classical SPS system in $L^{2}\left(\mathbb{R}^{3}\right)$, and in the energy space $H^{1}\left(\mathbb{R}^{3}\right)$. In the context of higher-order HartreeFock equations, the existence and uniqueness of solutions in $L^{2}\left(\mathbb{R}^{3}\right), H^{2 J}\left(\mathbb{R}^{3}\right)$ and $H^{J}\left(\mathbb{R}^{3}\right)$ is proved for $J \geq 2$ in [6]. Theorem 1.1 extends this result to the case of the SPS system.

\section{Proof of Theorem 1.1}

In this section we prove Theorem 1.1 by proceeding in two steps. First, we collect a few technical lemmas providing necessary Lipschitz bounds to set a contraction argument and obtain the existence and uniqueness of solutions in $L^{q, p}(\gamma)$. The second step consists in the proof of the existence and uniqueness of solutions in $H^{2 J}(\gamma)$. From this point onward, $\kappa$ will denote variant universal constants that may change from line to line of an inequality, and we will emphasize it when it depends on important parameters. Also, we shall use the notation $\zeta(0)$ instead of $\zeta(t=0)$ for all functions, and $\Psi:=\psi-\varphi$ and $\rho_{\Psi}:=\rho_{\psi}-\rho_{\varphi}$ when there is no confusion.

For all $J \in \mathbb{N}^{\star}$, we denote by $U_{J}(t)$ the propagator of the free higher-order Schrödinger operator. More precisely,

$$
U_{J}(t)=e^{-\mathrm{i} t H_{J}}, \quad H_{J}:=-\sum_{j=0}^{J} \frac{\alpha(j) \hbar^{2 j}}{m^{2 j-1} c^{2 j-2}} \Delta^{j} .
$$

In particular, $U_{J}(t)$ is an unitary operator on every $H^{s}$ for all $s \in \mathbb{R}$ (see Ref. [6], Proposition 3.4). In order to prove the existence of solutions, we seek a fixed point of the following functional:

$$
\begin{aligned}
\Lambda[\psi](t, x):= & U_{J}(t) \phi \\
& \quad-\mathrm{i} \int_{0}^{t} U_{J}(t-s)\left(c_{c} \frac{1}{|x|} \psi+c_{p}\left(\rho_{\psi} \star \frac{1}{|x|}\right) \psi-c_{s} \rho_{\psi}^{\frac{1}{3}} \psi\right)(x, s) d s \\
:= & U_{J}(t) \phi-\mathrm{i} \int_{0}^{t} U_{J}(t-s)(\mathfrak{C}(\psi)+\mathfrak{P}(\psi)-\mathfrak{S}(\psi))(x, s) d s,
\end{aligned}
$$


in a well-chosen closed ball. To ease the notation, we shall use the following:

$$
\Lambda_{I}(\xi)(t, x):=\int_{I \cap\{s, t\}} U_{J}(t-s) \xi(s, x) d s
$$

for all time intervals $I$, so that we can write

$$
\Lambda[\psi](t, x):=U_{J}(t) \phi-\mathrm{i} \Lambda_{I}(\mathfrak{C}(\psi)+\mathfrak{P}(\psi)-\mathfrak{S}(\psi))(t, x)
$$

\section{$2.1 L^{q, p}(\gamma)$ solutions}

In this subsection, we prove the first part of Theorem 1.1. For that purpose, we need the following local Strichartz estimates for the propagator $U_{J}(t)$.

Lemma 2.1 ([21]) Let $J \geq 2,\left(q_{1}, p_{1}\right),\left(q_{2}, p_{2}\right) \in \mathcal{J}$, and I a finite time interval of length less than 1. Then:

1. There exists $\kappa:=\kappa\left(p_{1}\right)$ such that

$$
\left\|U_{J}(\cdot) \zeta\right\|_{L_{I}^{q_{1}} \cdot p_{1}(\gamma)} \leq \kappa\|\zeta\|_{L^{2}(\gamma)}, \quad \text { for all } \zeta \in L^{2}(\gamma) .
$$

2. If I contains the origin, there exists $\kappa:=\kappa\left(p_{1}, p_{2}\right)$ such that

$$
\left\|\Lambda_{I}(\zeta)\right\|_{L_{I}^{q_{1}, p_{1}}(\gamma)} \leq \kappa\|\zeta\|_{L_{I}^{\frac{q_{2}}{q_{2}-1},}, \frac{p_{2}}{p_{2}-1}(\gamma)}, \quad \text { for all } \zeta \in L^{\frac{q_{2}}{q_{2}-1}, \frac{p_{2}}{p_{2}-1}}(\gamma) .
$$

This lemma is an extension of the results of [21]. The adaptation of the classical Strichartz' estimates to our weighted spaces is straightforward, and we refer the reader to [17] for a proof. Thanks to Lemma 2.1, we have

$$
\left\|U_{J}(\cdot) \zeta\right\|_{\mathfrak{B}_{I}^{q_{1}, p_{1}}(\gamma)} \leq \kappa\left(p_{1}\right)\|\zeta\|_{L^{2}(\gamma)}, \quad\left\|\Lambda_{I}(\zeta)\right\|_{\mathfrak{B}_{I}^{q_{1}, p_{1}}} \leq \kappa\left(p_{1}, p_{2}\right)\|\zeta\|_{\mathfrak{B}_{I}^{\prime q_{2}, p_{2}}(\gamma)},
$$

whenever the right hand side is finite. Next, we recall the following mass-conservation property of the SPS system.

Lemma 2.2 Let $T>0$ and $\phi=\left(\phi_{i}\right)_{i \in \mathbb{N}}$ a set of initial data in $L^{2}(\gamma)$. If there exists a weak solution $\psi(t)=\left(\psi_{i}(t)\right)_{i \in \mathbb{N}}$ to the SPS system on $[0, T]$, then $\left(\psi_{i}(t)\right)_{i \in \mathbb{N}}$ is such that $\|\psi(t)\|_{L^{2}(\gamma)}=\|\phi\|_{L^{2}(\gamma)}$ for all $t \in[0, T]$.

Proof On the one side, we multiply the SPS system (1) by $\bar{\psi}_{l}$ and integrate with respect to space. On the opposite side, we replace $k$ by $l$ in (1), take the complex conjugate of the system, multiply by $\phi_{l}$, integrate with respect to space, and integrate by parts the term $-\sum_{j=0}^{J} \frac{\alpha(j) \hbar^{2 j}}{m^{2 j-1} c^{2 j-2}} \int_{\mathbb{R}^{3}}\left[\Delta^{j} \bar{\psi}_{l}\right] \psi_{k} d x$. Eventually, summing up the two equalities leads to the desired the result.

Now, we estimate the linear and nonlinear parts in $\mathfrak{B}_{T}^{q, p}(\gamma)$ for all $(q, p) \in \mathfrak{J}$ and $T>0$. First, we have

Lemma 2.3 Let $T>0,(q, p) \in \mathfrak{J}$ such that $3<p \leq 6$ and $\psi, \varphi \in \mathfrak{B}_{T}^{q, p}(\gamma)$. Then there exists $\kappa:=\kappa(p)>0$ such that

$$
\|\mathfrak{C}(\Psi)\|_{\mathfrak{B}_{T}^{\prime q, p}(\gamma)} \leq \kappa \max \left(T, T^{1-\frac{3}{J}\left(\frac{1}{2}-\frac{2}{p}\right)}\right)\|\Psi\|_{\mathfrak{B}_{T}^{q, p}(\gamma)} .
$$


Proof The proof is based on a cut-off of the Coulomb potential $\frac{1}{|x|}$. Indeed, for all function $\chi \in C^{\infty}$ such that $\chi(r)=1$ for $0 \leq r \leq 1$ and $\chi(r)=0$ for $r \geq 2$, we have clearly

$$
\frac{1}{|x|}=\frac{1}{|x|}(1-\chi(|r|))+\frac{1}{|x|} \chi(|x|) \in L^{a}+L^{b} \quad \text { for all }(a, b) \in\left[\frac{3}{2}, 3[\times] 3,+\infty\right] .
$$

On the one side, we set $b=+\infty$ and obtain

$$
\|\mathfrak{C}(\Psi) \chi(|x|)\|_{L_{T}^{1,2}(\gamma)} \leq\left\|\frac{\chi(|x|)}{|x|}\right\|_{L_{x}^{\infty}} T\|\Psi\|_{L_{T}^{\infty, 2}(\gamma)} \leq \kappa T\|\Psi\|_{L_{T}^{\infty, 2}(\gamma)} .
$$

On the opposite side, using Hölder's inequality in time and space, combined with the fact that $(q, p) \in \mathfrak{J}$, we obtain

$$
\|\mathfrak{C}(\Psi)(1-\chi(|x|))\|_{L_{T}^{\frac{q}{q-1}, \frac{p}{p-1}(\gamma)}} \leq T^{1-\frac{3}{J}\left(\frac{1}{2}-\frac{2}{p}\right)}\left\|\frac{\chi(|x|)}{|x|}\right\|_{L_{x}^{\frac{p}{p-2}}}\|\Psi\|_{L_{T}^{q, p}(\gamma)} .
$$

The right hand side of this estimate is finite for all $3<p \leq 6$, which ensures that $\frac{p}{p-2} \in\left[\frac{3}{2}, 3[\right.$. Summing up the two inequalities we get (4).

Now, let us make clear the relation between the norms of the density $\rho_{\psi}$ and the wavefunction $\psi$. Thanks to Minkowski's inequality, we have $\|\psi\|_{L^{p}(\gamma)} \leq\left\|\rho_{\psi}^{\frac{1}{2}}\right\|_{L^{p}}$ for all $p \leq 2$. Also, using the reverse Minkowski inequality for all $p \geq 2$, we obtain $\left\|\rho_{\psi}^{\frac{1}{2}}\right\|_{L^{p}} \leq\|\psi\|_{L^{p}(\gamma)}$. We shall use this property tacitly in the sequel. Now, the Poisson part of the system satisfies the following.

Lemma 2.4 Let $T>0,(q, p) \in \mathfrak{J}$ such that $3<p \leq 6$ and $\psi, \varphi \in \mathfrak{B}_{T}^{q, p}(\gamma)$. Then there exists a constant $\kappa:=\kappa(p)$ such that

$$
\begin{aligned}
& \|\mathfrak{P}(\psi)-\mathfrak{P}(\varphi)\|_{\mathfrak{B}_{T}^{\prime q, p}(\gamma)} \\
& \quad \leq \kappa\left(\|\psi\|_{L^{\infty, 2}(\gamma)}+\|\varphi\|_{L^{\infty, 2}(\gamma)}\right)^{2} \max \left(T, T^{1-\frac{3}{J}\left(\frac{1}{2}-\frac{2}{p}\right)}\right)\|\Psi\|_{\mathfrak{B}_{T}^{q, p}(\gamma)} .
\end{aligned}
$$

Proof The proof is the same as in Ref. [17], we sketch it here for the reader's convenience. We have

$$
\|\mathfrak{P}(\psi)-\mathfrak{P}(\varphi)\|_{\mathfrak{B}_{T}^{\prime q, p}(\gamma)} \leq\left\|\left(\rho_{\Psi} \star \frac{1}{|x|}\right) \varphi\right\|_{\mathfrak{B}_{T}^{\prime q, p}(\gamma)}+\left\|\left(\rho_{\psi} \star \frac{1}{|x|}\right) \Psi\right\|_{\mathfrak{B}_{T}^{\prime q, p}(\gamma)} .
$$

We shall use the cut-off of the Coulomb potential given in the proof of Lemma 2.3. On the one hand, recalling that $\rho_{\psi}=\sum_{i} \gamma_{i}\left|\psi_{i}\right|^{2}$ and using Young's and Hölder's inequalities, we get

$$
\left\|\left(\rho_{\Psi} \star \frac{\chi(|x|)}{|x|}\right) \varphi\right\|_{L_{T}^{1,2}(\gamma)} \leq \kappa\|\varphi\|_{L^{\infty, 2}(\gamma)}\left(\|\psi\|_{L^{\infty, 2}(\gamma)}+\|\varphi\|_{L^{\infty, 2}(\gamma)}\right) T\|\Psi\|_{L_{T}^{\infty, 2}(\gamma)},
$$


and

$$
\begin{aligned}
& \left\|\left(\rho_{\Psi} \star \frac{1-\chi(|x|)}{|x|}\right) \varphi\right\|_{L_{T}^{\frac{q}{q-1}, \frac{p}{p-1}}(\gamma)} \\
& \quad \leq \kappa\|\varphi\|_{L^{\infty, 2}(\gamma)}\left(\|\psi\|_{L^{\infty, 2}(\gamma)}+\|\varphi\|_{L^{\infty, 2}(\gamma)}\right) T^{1-\frac{3}{J}\left(\frac{1}{2}-\frac{2}{p}\right)}\|\Psi\|_{L_{T}^{q, p}(\gamma)} .
\end{aligned}
$$

Equivalently, we have

$$
\left\|\left(\rho_{\psi} \star \frac{\chi(x)}{|x|}\right) \Psi\right\|_{L_{T}^{1,2}(\gamma)} \leq \kappa\|\psi\|_{L^{\infty, 2}(\gamma)}^{2} T\|\Psi\|_{L_{T}^{\infty, 2}(\gamma)},
$$

and

$$
\left\|\left(\rho_{\psi} \star \frac{1-\chi(x)}{|x|}\right) \Psi\right\|_{L_{T}^{\frac{q}{q-1}, \frac{p}{p-1}(\gamma)}} \leq \kappa\|\psi\|_{L^{\infty, 2}(\gamma)}^{2} T^{1-\frac{3}{J}\left(\frac{1}{2}-\frac{2}{p}\right)}\|\Psi\|_{L_{T}^{q, p}(\gamma)} .
$$

Gathering these estimates, we obtain (5). Observe that the condition $3<p \leq 6$ is needed to bound the short-range part of the potential as in Lemma 2.3.

Now, we provide estimates for the Slater part of the SPS system.

Lemma 2.5 Let $T>0,(q, p) \in \mathfrak{J}$ with $p>2$ and $\psi, \varphi \in \mathfrak{B}_{T}^{q, p}(\gamma)$. Then there exists a pair $(r, s) \in \mathfrak{J}$ such that

$$
\|\mathfrak{S}(\psi)-\mathfrak{S}(\varphi)\|_{L_{T}^{\frac{r}{r-1}, \frac{s}{s-1}(\gamma)}} \leq \kappa T^{1-\frac{1}{2 \jmath}}\left(\|\psi\|_{L_{T}^{q, p}(\gamma)}^{\frac{2}{3}}+\|\varphi\|_{L_{T}^{q, p}(\gamma)}^{\frac{2}{3}}\right)\|\Psi\|_{L_{T}^{\infty, 2}(\gamma)} .
$$

Proof We follow the argument of [20] using different $L^{q, p}(\lambda)$ spaces. First, using a firstorder Taylor expansion, we can write for all $\theta \in[0,1]$

$$
\begin{aligned}
\mathfrak{S}(\psi)-\mathfrak{S}(\varphi) & =\int_{0}^{1} \partial_{\zeta} \mathfrak{S}(\varphi+\theta \Psi) \cdot \Psi d \theta \\
& =\frac{2}{3} \int_{0}^{1} \mathfrak{R}\left(\langle\varphi+\theta \Psi, \Psi\rangle_{\ell^{2}(\gamma)}\right) \rho_{\varphi+\theta \Psi}^{-\frac{2}{3}}(\varphi+\theta \Psi) d \theta \\
& +\int_{0}^{1} \rho_{\varphi+\theta \Psi}^{\frac{1}{3}} \Psi d \theta:=\mathfrak{T}_{1}+\mathfrak{T}_{2} .
\end{aligned}
$$

Indeed, the Fréchet derivative of $\rho_{\zeta}^{\frac{1}{3}} \zeta$ is given by

$$
\partial_{\psi}\left(\rho_{\psi}^{\frac{1}{3}} \psi\right) h=\frac{2}{3} \Re\langle h, \psi\rangle_{\ell^{2}(\gamma)} \rho_{\psi}^{-\frac{2}{3}} \psi+\rho_{\psi}^{\frac{1}{3}} h
$$

where the symbol $\Re$ denotes the real part of complex numbers, and $\langle h, \psi\rangle_{\ell^{2}(\gamma)}$ denotes the Euclidean scalar product in $\ell^{2}(\gamma),\langle h, \psi\rangle_{\ell^{2}(\gamma)}:=\sum_{j} \gamma_{j} h_{j} \bar{\psi}_{j}$. Our aim is to estimate $\mathfrak{T}_{1}$ and $\mathfrak{T}_{2}$ in $L^{\frac{p}{p-1}}, \frac{r}{r-1}(\gamma)$. The proof of this fact is a straightforward application of Minkowski's inequality. Indeed, let $(q, p) \in \mathfrak{J}$, then using Hölder's inequality in space and time we 
get

$$
\begin{aligned}
& \left\|\mathfrak{T}_{2}\right\|_{L_{T}^{\frac{J p}{J p-1}, \frac{6 p}{3 p+4}(\gamma)}} \leq\left\|\rho_{\varphi+\theta \Psi}^{\frac{1}{3}}\right\|_{L_{T}^{\frac{J p}{J p-1}, \frac{3}{2} p}}\|\Psi\|_{L_{T}^{\infty, 2}(\gamma)} \\
& =\left\|\rho_{\varphi+\theta \Psi}^{\frac{1}{2}}\right\|_{L_{T}^{\frac{2}{3} \frac{J p}{p-1}, p}}^{\frac{2}{3}}\|\Psi\|_{L_{T}^{\infty, 2}(\gamma)} \\
& \leq\|\varphi+\theta \Psi\|_{L_{T}^{\frac{2}{3}} \frac{\frac{J p}{3 p-1}, p}{3}(\gamma)}^{\frac{L^{2}}{3}}\|\Psi\|_{L_{T}^{\infty, 2}(\gamma)} \\
& \leq T^{1-\frac{1}{2)}}\left(\|\varphi\|_{L^{q, p}(\gamma)}^{\frac{2}{3}}+\|\psi\|_{L^{q, p}(\gamma)}^{\frac{2}{3}}\right)\|\Psi\|_{L_{T}^{\infty, 2}(\gamma)} .
\end{aligned}
$$

In order to pass from the second to the third line of the estimate above, we used the fact that $\left\|\rho_{\zeta}^{\frac{1}{2}}\right\|_{L^{q, p}} \leq\|\zeta\|_{L^{q, p}(\gamma)}$ for all $p \geq 2$ and $\zeta \in L^{q, p}(\gamma)$. Observe that $\frac{J p}{J p-1}$ is the conjugate of $J p$ and $\frac{6 p}{3 p+4}$ is the conjugate of $\frac{6 p}{3 p-4}$, and that the pair $\left(J p, \frac{6 p}{3 p-4}\right) \in \mathfrak{J}$. Eventually, we have obviously $1-\frac{1}{2 J}>0$ for all $J \geq 2$. Now, we make an estimate of $\mathfrak{T}_{1}$ :

$$
\begin{aligned}
& \left\|\mathfrak{T}_{1}\right\|_{L_{T}^{\frac{J p}{p-1}, \frac{6 p}{3 p+4}}(\gamma)} \leq \eta\left\|\rho_{\varphi+\theta \Psi}^{\frac{1}{2}} \rho_{\Psi}^{\frac{1}{2}} \rho_{\varphi+\theta \Psi}^{-\frac{2}{3}}|\varphi+\theta \Psi|\right\|_{L_{T}^{\frac{J p}{j p-1}}, \frac{6 p}{3 p+4}(\gamma)} \\
& \leq \eta\left\|\rho^{\frac{1}{2}} \quad\right\| \frac{\frac{I p}{2}}{\left[\rho_{\varphi+\theta \Psi}^{-\frac{1}{6}} \rho_{\Psi}^{\frac{1}{2}}|\varphi+\theta \Psi|\right]} L_{T}^{\frac{6 p}{T p-1}, \frac{3 p+4}{3 p+4}} \\
& =\eta\left\|\rho_{\varphi+\theta \Psi}^{-\frac{1}{6}} \rho_{\Psi}^{\frac{1}{2}} \rho_{\varphi+\theta \Psi}^{\frac{1}{2}}\right\|_{L_{T}^{J p-1}, \frac{6 p}{3 p+4}} \\
& \leq \eta\left\|\rho_{\varphi+\theta \Psi}^{\frac{1}{3}}\right\|_{L_{T}^{\frac{J p}{p-1}, \frac{3}{2} p}}\left\|\rho_{\Psi}^{\frac{1}{2}}\right\|_{L_{T}^{\infty, 2}} \\
& \leq \eta T^{1-\frac{1}{2 J}}\left(\|\varphi\|_{L^{q, p}(\gamma)}^{\frac{2}{3}}+\|\psi\|_{L^{q, p}(\gamma)}^{\frac{2}{3}}\right)\|\Psi\|_{L_{T}^{\infty, 2}(\gamma)} .
\end{aligned}
$$

In the first line, we used the Cauchy-Schwartz inequality, $\left|\langle h, \psi\rangle_{\ell^{2}(\gamma)}\right|^{2} \leq \rho_{\psi} \rho_{h}$ and the notation $|\zeta|=\left(\left|\zeta_{j}\right|\right)_{j \in \mathbb{N}}$. In the second line, we used the fact that $\|\zeta\|_{L^{q, p}(\gamma)} \leq\left\|\rho_{\zeta}^{\frac{1}{2}}\right\|_{L^{q, p}}$ for all $p \leq 2$ and $\zeta \in L^{q, p}(\gamma)$. Clearly, we have $\frac{6 p}{3 p+4} \leq 2$. Eventually, the proof of the lemma is achieved by setting $(r, s)=\left(J p, \frac{6 p}{3 p-4}\right)$.

Now, we are able to set up a contraction argument to prove the first part of Theorem 1.1. Let $0<T \leq 1$ to be fixed later on, $\phi=\left(\phi_{i}\right)_{i \in \mathbb{N}}$ be a set of orthonormal initial data in $L^{2}(\gamma)$, and $\psi=\left(\psi_{i}\right)_{i \in \mathbb{N}}, \varphi=\left(\varphi_{i}\right)_{i \in \mathbb{N}}$ in the following closed ball:

$$
B_{R}:=\left\{\zeta \in \mathfrak{B}_{T}^{q, p}(\gamma) \cap L^{\infty}\left([0, T] ; L^{2}(\gamma)\right),\|\zeta\|_{\mathfrak{B}_{T}^{q, p}(\gamma)} \leq R\right\} .
$$

Using Lemmas 2.1-2.5, we can write for all $(q, p) \in \mathfrak{J}$ such that $3<p \leq 6$ and $J \geq 2$, We have

$$
\begin{aligned}
\| \Lambda[\psi](t, x) & \|_{\mathfrak{B}_{T}^{q, p}(\gamma)} \\
\leq & \kappa(p)\|\phi\|_{L^{2}(\gamma)} \\
& \quad+\kappa(p) \max \left(T, T^{1-\frac{3}{J}\left(\frac{1}{2}-\frac{2}{p}\right)}, T^{1-\frac{1}{2 J}}\right)\left(1+\|\phi\|_{L^{2}(\gamma)}^{2}+\|\psi\|_{L_{T}^{q, p}(\gamma)}^{\frac{2}{3}}\right)\|\psi\|_{\mathfrak{B}_{T}^{q, p}(\gamma)} .
\end{aligned}
$$


Now, since $\frac{3}{J}\left(\frac{1}{2}-\frac{2}{p}\right) \leq \frac{1}{2 J}$, we let $R:=2 \kappa(p)\|\phi\|_{L^{2}(\gamma)}$ and

$$
T \leq \min \left\{1,\left[2 \kappa(p)\left(1+4 \kappa^{2}(p)\|\phi\|_{L^{2}(\gamma)}^{2}+\left(2 \kappa(p)\|\phi\|_{L^{2}(\gamma)}\right)^{\frac{2}{3}}\right)\right]^{-\frac{2 J p}{2 / p-3 p+6}}\right\} .
$$

Therefore, we get

$$
\begin{aligned}
& \|\Lambda[\psi](t, x)\|_{\mathfrak{B}_{T}^{q, p}(\gamma)} \\
& \quad \leq \frac{R}{2}+\kappa(p) T^{1-\frac{3}{J}\left(\frac{1}{2}-\frac{2}{p}\right)}\left(1+4 \kappa^{2}(p)\|\phi\|_{L^{2}(\gamma)}^{2}+\left(2 \kappa(p)\|\phi\|_{L^{2}(\gamma)}\right)^{\frac{2}{3}}\right) R \leq R .
\end{aligned}
$$

This shows that $\Lambda$ maps $B_{R}$ into itself. Equivalently,

$$
\begin{aligned}
& \|\Lambda[\psi](t, x)-\Lambda[\varphi](t, x)\|_{\mathfrak{B}_{T}^{q, p}(\gamma)} \\
& \leq \quad \kappa(p) \max \left(T, T^{1-\frac{3}{J}\left(\frac{1}{2}-\frac{2}{p}\right)}, T^{1-\frac{1}{2 J}}\right) \\
& \quad \times\left(1+2\|\phi\|_{L^{2}(\gamma)}^{2}+\left(\|\psi\|_{L_{T}^{q, p}(\gamma)}^{\frac{2}{3}}+\|\varphi\|_{L_{T}^{q, p}(\gamma)}\right)^{\frac{2}{3}}\right)\|\Psi\|_{\mathfrak{B}_{T}^{q, p}(\gamma)} .
\end{aligned}
$$

Therefore, if we now let

$$
T \leq \min \left\{1,\left[4 \kappa(p)\left(1+4 \kappa^{2}(p)\|\phi\|_{L^{2}(\gamma)}^{2}+\left(2 \kappa(p)\|\phi\|_{L^{2}(\gamma)}\right)^{\frac{2}{3}}\right)\right]^{-\frac{2 J p}{2 / p-3 p+6}}\right\}
$$

then there exists a constant $0<\tilde{\eta}<1$ such that

$$
\|\Lambda[\psi](t, x)-\Lambda[\varphi](t, x)\|_{\mathfrak{B}_{T}^{q, p}(\gamma)} \leq \tilde{\eta}\|\psi-\varphi\|_{\mathfrak{B}_{T}^{q, p}(\gamma)} .
$$

We infer that $\Lambda$ is a strict contraction on $B_{R}$. Therefore, the SPS system has a solution in $L^{\infty}\left([0, T] ; L^{2}(\gamma)\right)$. Moreover, the solution is unique in $L^{\infty}\left([0, T] ; L^{2}(\gamma)\right) \cap L_{T}^{q, p}$ for all $(q, p) \in \mathfrak{J}$ such that $3<p \leq 6$. The fact that the solution is in $C^{0}\left([0, T] ; L^{2}(\gamma)\right)$ follows using standard arguments. The last point to make clear is the global existence of the solution, which is a consequence of Lemma 2.2. Indeed, the time $T>0$ fixed above depends only on the $L^{2}$ norm of the initial data, which is conserved by the dynamics of the SPS system. Thus, one can reiterate the contraction argument to cover the whole real line.

\section{$2.2 H^{2 J}(\gamma)$ solutions}

In this section we prove the second part of Theorem 1.1. For that purpose, we follow the method of Kato (see Refs. [22, 23]) by setting a fixed point argument. From this point onward, we shall use the notation $C_{T}^{p} \mathbf{X}:=C^{p}([0, T] ; \mathbf{X}), L_{T}^{p} \mathbf{X}:=L^{p}([0, T] ; \mathbf{X})$ and $W_{T}^{q, p} \mathbf{X}:=$ $W^{q, p}([0, T] ; \mathbf{X})$ for a given functional space $\mathbf{X}$. Let us summarize the following properties associated with the operator $U_{J}(t)$.

Lemma 2.6 Let $J \geq 2,(q, p) \in \mathfrak{J}$ and $\zeta \in H^{2 J}(\gamma)$, then for all $t \in[0, T]$ we have $U_{J}(t) \zeta \in$ $C_{T}^{0} H^{2 J}(\gamma)$ and $\left\|U_{J}(t) \zeta\right\|_{L_{T}^{\infty} H^{2 J}(\gamma)} \leq\|\zeta\|_{H^{2 J}(\gamma)}$. Moreover, $U_{J}(t) \zeta \in C_{T}^{1} L^{2}(\gamma) \cap W_{T}^{1, q} L^{p}(\gamma)$ and there exists a constant $\kappa=: \kappa(p)$ such that $\left\|U_{J}(t) \zeta\right\|_{W_{T}^{1, q} L^{p}(\gamma)} \leq \kappa\|\zeta\|_{H^{2 J}(\gamma)}$. 
Proof The first assertion follows immediately from the expression of $U_{J}(t)$ in Fourier variables. Now, let $\zeta$ be an initial data to the equation

$$
i \partial_{t} \psi=\sum_{j=1}^{J}(-1)^{j+1} \frac{\alpha(j) \hbar^{2 j}}{m^{2 j-1} c^{2 j-2}}(-\Delta)^{j} \psi
$$

Then the second assertion is a consequence of Strichartz' estimates given in Lemma 2.1 and the fact that

$$
\partial_{t} \psi=\mathrm{i} \sum_{j=1}^{J}(-1)^{j} \frac{\alpha(j) \hbar^{2 j}}{m^{2 j-1} c^{2 j-2}}(-\Delta)^{j} \psi=\mathrm{i} U_{J}(t) \sum_{j=1}^{J}(-1)^{j} \frac{\alpha(j) \hbar^{2 j}}{m^{2 j-1} c^{2 j-2}}(-\Delta)^{j} \zeta .
$$

This finishes the proof.

This lemma provides an estimate which is equivalent to the first estimate in (3). The equivalent of the second estimate in (3) is a direct consequence of the following.

Lemma 2.7 Let $J \geq 2,\left(q_{1}, p_{1}\right),\left(q_{2}, p_{2}\right) \in \mathfrak{J}$ and $\zeta \in L_{T}^{\infty, 2}(\gamma)$ such that $\partial_{t} \zeta \in L_{T}^{\frac{q_{2}}{q_{2}-1}, \frac{p_{2}}{p_{2}-1}}(\gamma)$. Then $\Lambda_{[0, T]}(\zeta) \in L_{T}^{\infty} H^{2 J}(\gamma) \cap C_{T}^{1} L^{2}(\gamma) \cap W_{T}^{1, q_{1}} L^{p_{1}}(\gamma)$ and

$$
\begin{aligned}
& \left\|\Lambda_{[0, T]}(\zeta)\right\|_{L_{T}^{q_{1}, p_{1}}(\gamma)} \leq \kappa\left(p_{1}\right)\|\zeta\|_{L_{T}^{1,2}(\gamma)}, \\
& \left\|\partial_{t} \Lambda_{[0, T]}(\zeta)\right\|_{L_{T}^{q_{1}, p_{1}}(\gamma)} \leq \kappa\left(p_{1}\right)\|\zeta(0)\|_{L^{2}(\gamma)}+\kappa\left(p_{1}, p_{2}\right)\left\|\partial_{t} \zeta\right\|_{L_{T}^{\frac{q_{2}}{q_{2}-1}, \frac{p_{2}}{p_{2}-1}}(\gamma)}, \\
& \left\|(-\Delta)^{J} \Lambda_{[0, T]}(\zeta)\right\|_{L_{T}^{\infty, 2}(\gamma)} \leq\|\zeta\|_{L_{T}^{\infty, 2}(\gamma)}+\kappa\|\zeta(0)\|_{L^{2}(\gamma)}+\kappa\left(p_{2}\right)\left\|\partial_{t} \zeta\right\|_{L_{T}^{\frac{q_{2}}{q_{2}-1}, p_{2}-1}(\gamma)} .
\end{aligned}
$$

Moreover, if $\zeta \in C_{T}^{0} L^{2}(\gamma)$, then $\Lambda_{[0, T]}(\zeta) \in C_{T}^{0} H^{2 J}(\gamma)$.

Proof The proof is based on Strichartz' estimates given in Lemma 2.1. Indeed, the first assertion is nothing but the second estimate in Lemma 2.1 with the pair $(1,2)$ as the conjugate of $(\infty, 2) \in \mathfrak{J}$. To prove the second assertion, we observe that

$$
\partial_{t} \Lambda_{[0, T]}(\zeta)=\Lambda_{[0, T]}\left(\partial_{t} \zeta\right)+U_{J}(t) \zeta(0)
$$

This formula is justified by the fact that $\partial_{t} \zeta \in \mathfrak{B}_{T}^{\prime q_{2}, p_{2}} \subset L_{T}^{1} H^{-J}(\gamma)$, thus $\zeta \in C_{T}^{0} H^{-J}(\gamma)$. In particular $\zeta(0)$ makes sense, actually it belongs to $L^{2}(\gamma)$. Thanks to Lemma 2.1, we have

$$
\left\|\partial_{t} \Lambda_{[0, T]}(\zeta)\right\|_{L_{T}^{q_{1}, p_{1}}} \leq \kappa(p)\left\|\partial_{t} \zeta\right\|_{\mathfrak{L}_{T}^{\frac{q_{2}}{q_{2}}, \frac{p_{2}}{p_{2}-I}}}+\kappa\|\zeta(0)\|_{L^{2}(\gamma)} .
$$

In order to prove the last assertion, we write

$$
(-\Delta)^{J} \Lambda_{[0, T]}(\zeta)=\mathrm{i}\left(-\partial_{t} \Lambda_{[0, T]}(\zeta)+\zeta\right)
$$

This formulation is justified by the same argument as above, thus we have $(-\Delta)^{J} \Lambda_{[0, T]}(\zeta) \in$ $L_{T}^{\infty, 2}(\gamma)$, and the desired estimate follows immediately using the second assertion. 
Now, we have $\mathfrak{C}(\psi)+\mathfrak{P}(\psi)-\mathfrak{S}(\psi): H^{2 J}(\gamma) \rightarrow L^{2}$. Thus, it is rather easy to see that there exists $0 \leq s<2 J$ such that $\mathfrak{C}(\psi)+\mathfrak{P}(\psi)-\mathfrak{S}(\psi) \in C^{0}\left(H^{s}(\gamma), L^{2}(\gamma)\right)$. For instance, if we fix $s \geq 1$, we have

$$
\begin{aligned}
& \|\mathfrak{C}(\psi)\|_{L^{2}(\gamma)} \leq 2\|\nabla \psi\|_{L^{2}(\gamma)} \leq 2\|\psi\|_{H^{s}(\gamma)}, \\
& \|\mathfrak{P}(\psi)\|_{L^{2}(\gamma)} \leq 2\|\nabla \psi\|_{L^{2}(\gamma)}\|\psi\|_{L^{2}(\gamma)}^{2} \leq 2\|\psi\|_{H^{s}(\gamma)}^{3}, \\
& \|\mathfrak{S}(\psi)\|_{L^{2}(\gamma)} \leq\|\psi\|_{L^{2}(\gamma)}^{\frac{2}{3}}\|\psi\|_{L^{6}(\gamma)} \leq\|\psi\|_{H^{s}(\gamma)}^{\frac{5}{3}} .
\end{aligned}
$$

That is, $\mathfrak{C}(\psi)+\mathfrak{P}(\psi)-\mathfrak{S}(\psi) \in L_{T}^{\infty, 2}(\gamma)$ for all $\psi \in L_{T}^{\infty} H^{s}(\gamma)$. Also, there exists a continuous function $\eta: \mathbb{R}_{+}^{\star} \rightarrow \mathbb{R}_{+}^{\star}$ such that, for every $\psi \in L_{T}^{\infty} H^{s}(\gamma)$ satisfying $\|\psi\|_{L_{T}^{\infty} H^{s}(\gamma)} \leq K$, we have

$$
\|\mathfrak{C}(\psi)+\mathfrak{P}(\psi)-\mathfrak{S}(\psi)\|_{L_{T}^{\infty, 2}(\gamma)} \leq \eta(K)
$$

Let us write $\mathfrak{C}=\mathfrak{C}_{1}+\mathfrak{C}_{2}$, and equivalently for $\mathfrak{P}$ with respect to the short and long ranges of $\frac{1}{|x|}$. Then we have the following.

Lemma 2.8 Let $T>0,3<\bar{p} \leq 6,2<\tilde{p}<6$, and $\bar{q}, \tilde{q}$ such that $(\bar{q}, \bar{p}),(\tilde{q}, \tilde{p}) \in \mathfrak{J}$. Then, for all $\psi \in L_{T}^{\infty} H^{s}(\gamma)$ such that $\|\psi\|_{L_{T}^{\infty} H^{s}(\gamma)} \leq K$ and $\partial_{t} \psi \in L_{T}^{\bar{q}, \bar{p}}(\gamma) \cap L_{T}^{\infty, 2}(\gamma)$, we have

$$
\begin{aligned}
& \left\|\partial_{t} \mathfrak{C}_{1}(\psi)\right\|_{L_{T}^{1,2}(\gamma)} \leq \kappa T\left\|\partial_{t} \psi\right\|_{L_{T}^{\infty, 2}(\gamma)}, \\
& \left\|\partial_{t} \mathfrak{C}_{2}(\psi)\right\|_{L_{T}^{\frac{\bar{q}}{q}-\frac{\bar{p}}{p-1}}(\gamma)} \leq \kappa T^{1-\frac{3}{J}\left(\frac{1}{2}-\frac{1}{\bar{p}}\right)}\left\|\partial_{t} \psi\right\|_{L_{T}^{\bar{q}, \bar{p}}(\gamma)^{\prime}}, \\
& \left\|\partial_{t} \mathfrak{P}_{1}(\psi)\right\|_{L_{T}^{1,2}(\gamma)} \leq \kappa K^{2} T\left\|\partial_{t} \psi\right\|_{L_{T}^{\infty, 2}(\gamma)}, \\
& \left\|\partial_{t} \mathfrak{P}_{2}(\psi)\right\|_{L^{\frac{\bar{q}}{q-1}, \frac{\bar{p}}{p-1}}(\gamma)} \leq \kappa K^{2} T^{1-\frac{3}{J}\left(\frac{1}{2}-\frac{1}{\bar{p}}\right)}\left\|\partial_{t} \psi\right\|_{L_{T}^{\bar{q}, \bar{p}}(\gamma)}, \\
& \left\|\partial_{t} \mathfrak{S}(\psi)\right\|_{L^{\frac{\tilde{q}}{q-1}, \frac{\tilde{p}}{\tilde{p}-1}(\gamma)}} \leq \kappa K^{\frac{2}{3}} T^{1-\frac{3}{2 J}\left(\frac{1}{2}-\frac{1}{\tilde{p}}\right)}\left\|\partial_{t} \psi\right\|_{L_{T}^{\infty, 2}(\gamma)} .
\end{aligned}
$$

Proof Thanks to Lemmas 2.3-2.5, if $\psi, \varphi \in H^{2 J}(\gamma)$ such that $\|\psi\|_{H^{s}(\gamma)},\|\psi\|_{H^{s}(\gamma)} \leq K$, then for all $3<\bar{p} \leq 6$ and $2<\tilde{p}<6$ we have

$$
\begin{aligned}
& \left\|\mathfrak{C}_{1}(\psi)-\mathfrak{C}_{1}(\varphi)\right\|_{L^{2}(\gamma)} \leq \kappa\|\Psi\|_{L^{2}(\gamma)}, \\
& \left\|\mathfrak{C}_{2}(\psi)-\mathfrak{C}_{2}(\varphi)\right\|_{L^{\frac{\tilde{p}}{p-1}}(\gamma)} \leq \kappa\|\Psi\|_{L^{\bar{p}}(\gamma)}, \\
& \left\|\mathfrak{P}_{1}(\psi)-\mathfrak{P}_{1}(\varphi)\right\|_{L^{2}(\gamma)} \leq \kappa K^{2}\|\Psi\|_{L^{2}(\gamma)}, \\
& \left\|\mathfrak{P}_{2}(\psi)-\mathfrak{P}_{2}(\varphi)\right\|_{L^{\frac{\bar{p}}{p-1}}(\gamma)} \leq \kappa K^{2}\|\Psi\|_{L^{\bar{p}}(\gamma)}, \\
& \|\mathfrak{S}(\psi)-\mathfrak{S}(\varphi)\|_{L^{\frac{\tilde{p}}{p-1}}(\gamma)} \leq \kappa K^{\frac{2}{3}}\|\Psi\|_{L^{2}(\gamma)} .
\end{aligned}
$$


Consequently, thanks to Proposition 1.3.12 of [23], we have

$$
\begin{aligned}
& \left\|\partial_{t} \mathfrak{C}_{1}(\psi)\right\|_{L_{T}^{1,2}(\gamma)} \leq \kappa\left\|\partial_{t} \psi\right\|_{L_{T}^{1,2}(\gamma)}, \\
& \left\|\partial_{t} \mathfrak{P}_{1}(\psi)\right\|_{L_{T}^{1,2}(\gamma)} \leq \kappa K^{2}\left\|\partial_{t} \psi\right\|_{L_{T}^{1,2}(\gamma)}, \\
& \left\|\partial_{t} \mathfrak{C}_{2}(\psi)\right\|_{L_{T}^{\frac{\bar{q}}{q-1}, \frac{\bar{p}}{p-1}(\gamma)}} \leq \kappa\left\|\partial_{t} \psi\right\|_{L_{T}^{\frac{\bar{q}}{q-1}, \bar{p}}(\gamma)}, \\
& \left\|\partial_{t} \mathfrak{P}_{2}(\psi)\right\|_{L^{\frac{\bar{q}}{q-1}, \frac{\bar{p}}{p-1}(\gamma)}} \leq \kappa K^{2}\left\|\partial_{t} \psi\right\|_{L_{T}^{\frac{\bar{q}}{q-1}, \bar{p}}(\gamma)}, \\
& \left.\| \partial_{t} \mathfrak{S}(\psi)\right)\left\|_{L_{\frac{\tilde{q}}{\bar{q}-1}, \frac{\tilde{p}}{\bar{p}-1}(\gamma)}} \leq \kappa K^{\frac{2}{3}}\right\| \partial_{t} \psi \|_{L_{T}^{\frac{\tilde{q}}{q}, 2}(\gamma)} .
\end{aligned}
$$

Eventually, applying Hölder's inequality in time finishes the proof.

Next, we set a fixed point argument and finish the proof of Theorem 1.1. First, observe that the SPS system (1) and the Duhamel formulation (2) are equivalents. Indeed, let $\psi \in L_{T}^{\infty} H^{s}(\gamma)$, then $(-\Delta)^{J} \psi \in L_{T}^{\infty} H^{s-2 J}(\gamma)$ since $(-\Delta)^{J} \psi \in \mathcal{L}\left(H^{s}, H^{s-2 J}\right)$. Moreover, for all $T>0, \mathfrak{C}(\psi)+\mathfrak{P}(\psi)-\mathfrak{S}(\psi): I \rightarrow L^{2}(\gamma)$ is measurable since $\mathfrak{C}(\psi)+\mathfrak{P}(\psi)-\mathfrak{S}(\psi) \in$ $C^{0}\left(H^{s}(\gamma), L^{2}(\gamma)\right)$ and bounded since it is bounded on bounded sets, thus $\mathfrak{C}(\psi)+\mathfrak{P}(\psi)-$ $\mathfrak{S}(\psi) \in L_{T}^{\infty, 2}(\gamma)$. Therefore, Eqs. (1) and (2) are well defined on $L^{2}(\gamma)$. The equivalence follows using the fact that $\left(U_{J}(t)\right)_{t \in \mathbb{R}}$ is a group of isometries on $L^{2}(\gamma)$.

Let $0 \leq s<2 J$ such that the three estimates (8) hold true, and $(q, p) \in \mathfrak{J}$ such that $p=$ $\max \{\bar{p}, \tilde{p}\}$ of Lemma 2.8. Also, let $T, K>0$ be fixed hereafter, and introduce the space

$$
\begin{aligned}
\mathcal{L}(\gamma):= & \left\{\psi \in L_{T}^{\infty} H^{s}(\gamma) \cap W_{T}^{1, \infty} L^{2}(\gamma) \cap W_{T}^{1, q} L^{r}(\gamma)\right. \text { such that } \\
& \left.\|\psi\|_{L_{T}^{\infty} H^{s}(\gamma)}+\|\psi\|_{W_{T}^{1, \infty} L^{2}(\gamma)}+\|\psi\|_{W_{T}^{1, q} L^{r}(\gamma)} \leq K \text { and } \psi(0)=\phi\right\} .
\end{aligned}
$$

Notice that $\mathcal{L}(\gamma) \neq \varnothing$ for all $\gamma>0$ since $\psi(t)=\varphi \in \mathcal{L}$ and that $(\mathcal{L}, d)$ is a complete metric

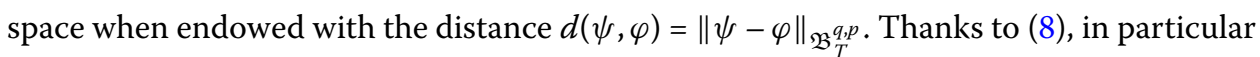
(9), we see that $\Lambda_{[0, T]}(\mathfrak{C}(\psi)+\mathfrak{P}(\psi)+\mathfrak{S}(\psi))(t, x)$ belongs to $C_{T}^{0} L^{2}(\gamma)$, therefore it is well defined. Now, thanks to Hölder's inequality, if $\psi \in \mathcal{L}(\gamma)$, then $\psi \in W_{T}^{1, \bar{q}} L^{\bar{p}}(\gamma)$. Indeed,

$$
\begin{aligned}
& \|\psi\|_{W_{T}^{1, \bar{q}} \overline{\bar{p}}(\gamma)}=\left(\|\psi\|_{L_{T}^{\bar{q}, \bar{p}}(\gamma)}^{2}+\left\|\partial_{t} \psi\right\|_{L_{T}^{\bar{q}, \bar{p}}(\gamma)}^{2}\right)^{\frac{1}{2}} \\
& \leq\left(\|\psi\|_{L_{T}^{\infty}(\gamma)}^{\frac{2(p-\bar{p})}{\bar{p}(p-2)}}\|\psi\|_{L_{T}^{\frac{q, p}{p(p-2)}}}^{2 \frac{p(\bar{p}-2)}{p(p)}}+\left\|\partial_{t} \psi\right\|_{L_{T}^{\infty}, 2(\gamma)}^{\frac{2(p-\bar{p})}{\bar{p}(p-2)}}\left\|\partial_{t} \psi\right\|_{L_{T}^{q, p}(\gamma)}^{2 \frac{p(\bar{p}-2)}{\bar{p}(p-2)}}\right)^{\frac{1}{2}} \\
& \leq K^{\frac{2(p-\bar{p})}{\bar{p}(p-2)}}\left(\|\psi\|_{L_{T}^{\frac{q, p}{p}(\gamma)}}^{\frac{p(\bar{p}-2)}{\bar{q}(p)}}+\left\|\partial_{t} \psi\right\|_{L_{T}^{q, p}(\gamma)}^{2 \frac{p(\bar{p}-2)}{p(p-2)}}\right)^{\frac{1}{2}} \leq K^{\frac{2(p-\bar{p})}{\bar{p}(p-2)}} K^{\frac{p(\bar{p}-2)}{\bar{p}(p-2)}}=K .
\end{aligned}
$$

With Lemma 2.8, one deduces the following estimates:

$$
\begin{aligned}
& \left\|\partial_{t} \mathfrak{C}_{1}(\psi)\right\|_{L_{T}^{1,2}(\gamma)} \leq \kappa K T, \quad\left\|\partial_{t} \mathfrak{C}_{2}(\psi)\right\|_{L_{T}^{\frac{\bar{q}}{q-1}, \frac{\bar{p}}{p-1}}(\gamma)} \leq \kappa K T^{1-\frac{3}{T}\left(\frac{1}{2}-\frac{1}{\bar{p}}\right)}, \\
& \left\|\partial_{t} \mathfrak{P}_{1}(\psi)\right\|_{L_{T}^{1,2}(\gamma)} \leq \kappa K^{3} T, \quad\left\|\partial_{t} \mathfrak{P}_{2}(\psi)\right\|_{L^{\frac{\bar{q}}{q-1}, \frac{\bar{p}}{p-1}(\gamma)}} \leq \kappa K^{3} T^{1-\frac{3}{J}\left(\frac{1}{2}-\frac{1}{\bar{p}}\right)}, \\
& \left.\| \partial_{t} \mathfrak{S}(\psi)\right) \|_{L^{\frac{\tilde{q}}{\bar{q}-1}, \frac{\tilde{p}}{\bar{p}-1}(\gamma)} \leq \kappa K^{\frac{5}{3}} T^{1-\frac{3}{2}\left(\frac{1}{2}-\frac{1}{\bar{p}}\right)} .}
\end{aligned}
$$


Next, using (9) with the first estimate of Lemma 2.7, we obtain the existence of a constant $\tilde{\kappa}$ independent of $K$ and $T$ such that

$$
\left\|\Lambda_{[0, T]}(\mathfrak{C}(\psi)+\mathfrak{P}(\psi)+\mathfrak{S}(\psi))\right\|_{L_{T}^{\infty, 2}(\gamma)} \leq \tilde{\kappa} T \eta(K)
$$

The other estimates of Lemma 2.7 show that $\Lambda_{[0, T]}(\mathfrak{C}(\psi)+\mathfrak{P}(\psi)+\mathfrak{S}(\psi)) \in L_{T}^{\infty} H^{2 J}(\gamma) \cap$ $W_{T}^{1, l} L^{m}(\gamma)$ for all $\psi \in \mathcal{L}(\gamma)$ and all pairs $(l, m) \in \mathfrak{J}$. Also, using Lemma 2.7 and estimates (9)-(10), we obtain (by possibly choosing a larger $\tilde{\kappa}$ )

$$
\begin{aligned}
\| \Lambda_{[0, T]} & (\mathfrak{C}(\psi)+\mathfrak{P}(\psi)+\mathfrak{S}(\psi)) \|_{W_{T}^{1, \infty} L^{2}(\gamma)} \\
& +\left\|\Lambda_{[0, T]}(\mathfrak{C}(\psi)+\mathfrak{P}(\psi)+\mathfrak{S}(\psi))\right\|_{W_{T}^{1, q} L^{p}(\gamma)} \\
\leq & \tilde{\kappa}\left(T \eta(K)+\left\|\mathfrak{C}_{1}(\phi)\right\|_{L^{2}(\gamma)}+\left\|\mathfrak{C}_{2}(\phi)\right\|_{L^{2}(\gamma)}+\left\|\mathfrak{P}_{1}(\phi)\right\|_{L^{2}(\gamma)}+\left\|\mathfrak{P}_{2}(\phi)\right\|_{L^{2}(\gamma)}\right. \\
& \left.+\|\mathfrak{S}(\phi)\|_{L^{2}(\gamma)}+\left[K+K^{3}\right]\left[T+T^{1-\frac{3}{T}\left(\frac{1}{2}-\frac{1}{\bar{p}}\right)}\right]+K^{\frac{5}{3}} T^{1-\frac{3}{2 J}\left(\frac{1}{2}-\frac{1}{\tilde{p}}\right)}\right),
\end{aligned}
$$

and

$$
\begin{aligned}
& \left\|\Lambda_{[0, T]}(\mathfrak{C}(\psi)+\mathfrak{P}(\psi)+\mathfrak{S}(\psi))\right\|_{L_{T}^{\infty} H^{2}(\gamma)} \\
& \leq \tilde{\kappa}\left([1+T] \eta(K)+\left\|\mathfrak{C}_{1}(\phi)\right\|_{L^{2}(\gamma)}+\left\|\mathfrak{C}_{2}(\phi)\right\|_{L^{2}(\gamma)}+\left\|\mathfrak{P}_{1}(\phi)\right\|_{L^{2}(\gamma)}+\left\|\mathfrak{P}_{2}(\phi)\right\|_{L^{2}(\gamma)}\right. \\
& \left.\quad+\|\mathfrak{S}(\phi)\|_{L^{2}(\gamma)}+\left[K+K^{3}\right]\left[T+T^{1-\frac{3}{J}\left(\frac{1}{2}-\frac{1}{\bar{p}}\right)}\right]+K^{\frac{5}{3}} T^{1-\frac{3}{2}\left(\frac{1}{2}-\frac{1}{\tilde{p}}\right)}\right)
\end{aligned}
$$

Combining these estimates with Lemma 2.6 and (10), we obtain (by possibly choosing $\operatorname{larger} \tilde{\kappa})$

$$
\begin{aligned}
& \|\Lambda[\psi]\|_{L_{T}^{\infty, 2}(\gamma)} \leq \tilde{\kappa}\left(\operatorname{Tr}(K)+\|\phi\|_{H^{2 J}(\gamma)}\right), \\
& \|\Lambda[\psi]\|_{W_{T}^{1, \infty} L^{2}(\gamma)}+\|\Lambda[\psi]\|_{W_{T}^{1, q} L^{p}(\gamma)} \\
& \leq \tilde{\kappa}\left(\|\phi\|_{H^{2 J}(\gamma)}+T \eta(K)+\left\|\mathfrak{C}_{1}(\phi)\right\|_{L^{2}(\gamma)}\right. \\
& \quad+\left\|\mathfrak{C}_{2}(\phi)\right\|_{L^{2}(\gamma)}+\left\|\mathfrak{P}_{1}(\phi)\right\|_{L^{2}(\gamma)}+\left\|\mathfrak{P}_{2}(\phi)\right\|_{L^{2}(\gamma)}+\|\mathfrak{S}(\phi)\|_{L^{2}(\gamma)} \\
& \left.\quad+\left[K+K^{3}\right]\left[T+T^{1-\frac{3}{T}\left(\frac{1}{2}-\frac{1}{p}\right)}\right]+K^{\frac{5}{3}} T^{1-\frac{3}{2 J}\left(\frac{1}{2}-\frac{1}{\tilde{p}}\right)}\right) .
\end{aligned}
$$

Also,

$$
\begin{aligned}
\|\Lambda[\psi]\|_{L_{T}^{\infty} H^{2}(\gamma)} \leq & \tilde{\kappa}\left(\|\phi\|_{H^{2 J}(\gamma)}+[1+T] \eta(K)+\left\|\mathfrak{C}_{1}(\phi)\right\|_{L^{2}(\gamma)}+\left\|\mathfrak{C}_{2}(\phi)\right\|_{L^{2}(\gamma)}\right. \\
& +\left\|\mathfrak{P}_{1}(\phi)\right\|_{L^{2}(\gamma)}+\left\|\mathfrak{P}_{2}(\phi)\right\|_{L^{2}(\gamma)}+\|\mathfrak{S}(\phi)\|_{L^{2}(\gamma)} \\
& \left.+\left[K+K^{3}\right]\left[T+T^{1-\frac{3}{J}\left(\frac{1}{2}-\frac{1}{\tilde{p}}\right)}\right]+K^{\frac{5}{3}} T^{1-\frac{3}{2 J}\left(\frac{1}{2}-\frac{1}{\tilde{p}}\right)}\right)
\end{aligned}
$$

Next, we choose $K$ in $\mathcal{L}(\gamma)$ such that

$$
\begin{aligned}
K= & 4 \tilde{\kappa}\left(\|\phi\|_{H^{2 J}(\gamma)}+\left\|\mathfrak{C}_{1}(\phi)\right\|_{L^{2}(\gamma)}+\left\|\mathfrak{C}_{2}(\phi)\right\|_{L^{2}(\gamma)}\right. \\
& \left.+\left\|\mathfrak{P}_{1}(\phi)\right\|_{L^{2}(\gamma)}+\left\|\mathfrak{P}_{2}(\phi)\right\|_{L^{2}(\gamma)}+\|\mathfrak{S}(\phi)\|_{L^{2}(\gamma)}\right),
\end{aligned}
$$


and

$$
T<\min \left\{1,\left[K\left(4 \tilde{\kappa}\left(\eta(K)+K+K^{3}+K^{\frac{5}{3}}\right)\right)^{-1}\right]^{\frac{22 \tilde{p}}{\bar{p}(2)-3)+6}}\right\} .
$$

Hence, using (12) we obtain

$$
\|\Lambda[\psi]\|_{W_{T}^{1, \infty} L^{2}(\gamma)}+\|\Lambda[\psi]\|_{W_{T}^{1, q} L^{p}(\gamma)}<\frac{1}{2} K
$$

Eventually, using the interpolation inequality $\|\psi\|_{H^{s}(\gamma)} \leq\|\psi\|_{L^{2}(\gamma)}^{\frac{2-s}{2}}\|\psi\|_{H^{2 J}(\gamma)}^{\frac{s}{2}}$, and the estimates (11) and (13), and possibly choosing $T$ smaller, we obtain

$$
\|\Lambda[\psi]\|_{L_{T}^{\infty} H^{s}(\gamma)}<\frac{1}{2} K
$$

In particular, the functional $\Lambda$ maps $\mathcal{L}(\gamma)$ into itself. A slight modification of the estimates of the nonlinear terms of the SPS system shows that one can choose $T$ possibly smaller in order to show that the mapping $\Lambda$ is a strict contraction on the complete metric $(\mathcal{L}(\gamma), d)$. Therefore, we can deduce the existence of a fixed point $\psi \in \mathcal{L}(\gamma)$ solving the system (1) with initial data $\phi \in H^{2 J}(\gamma)$. Lemma 2.6 and Lemma 2.7 allow one to deduce that $\psi(t, x) \in C_{T}^{0} H^{2 J}(\gamma) \cap C_{T}^{1} L^{2}(\gamma)$. For this purpose, the technical point is to show that $\mathfrak{C}(\psi)+\mathfrak{P}(\psi)-\mathfrak{S}(\psi) \in C_{T}^{0} L^{2}(\gamma)$. This can be achieved by observing that $\psi \in C_{T}^{0} L^{2}(\gamma)$ and using (13) to obtain $\psi \in L_{T}^{\infty} H^{2 J}(\gamma)$. Eventually, the interpolation inequality used before leads to $\psi \in C_{T}^{0} H^{s}(\gamma)$, hence $\mathfrak{C}(\psi)+\mathfrak{P}(\psi)-\mathfrak{S}(\psi) \in C_{T}^{0} L^{2}(\gamma)$ since $\mathfrak{C}(\psi)+\mathfrak{P}(\psi)-\mathfrak{S}(\psi) \in C\left(H^{s}(\gamma), L^{2}(\gamma)\right)$. Observe that $\psi \in W_{T}^{1, l} L^{m}(\gamma)$ for all pairs $(l, m) \in \mathfrak{J}$, and the uniqueness of the solution readily follows using a difference argument (the proof is in the same spirit of the one showing that the mapping $\Lambda$ is a strict contraction). The blow-up alternative and the continuous dependence on the initial data can be shown using standard arguments, and we refer to, e.g., [23]. Eventually, notice that the time $T$ depends on $J, p, \bar{p}, \tilde{p}$ and $K$, and that $K$ depends only on the initial data, therefore one can reiterate the argument (with initial data $\psi(T), \psi(2 T), \ldots$ ) and cover the whole real line, which finishes the proof of Theorem 1.1.

\section{$2.3 H^{J}(\gamma)$ solutions}

The proof of Corollary 1.2 can be achieved using a limiting procedure based on Theorem 1.1. Indeed, we construct a solution in $H^{J}(\gamma)$ as a limit of a sequence of solutions in $H^{2 J}(\gamma)$. The proof is standard, we skip it and refer to [23] for similar arguments (see also [20]). The key point is to show the boundedness of $\psi$ in $H^{J}(\gamma)$, which is a consequence of the following.

Lemma 2.9 Let $T>0$ and $\phi=\left(\phi_{i}\right)_{i \in \mathbb{N}}$ a set initial data in $H^{2 J}(\gamma)$ and $\psi(t)=\left(\psi_{i}(t)\right)_{i \in \mathbb{N}}$ the associated solution to the SPS system. Then, for all $t \in[0, T]$, we have

$$
\begin{aligned}
\mathcal{E}_{J}(\psi):= & -\sum_{k \in \mathbb{N}} \sum_{j=0}^{J}(-1)^{j} \frac{\alpha(j) \hbar^{2 j}}{m^{2 j-1} c^{2 j-2}} \int_{\mathbb{R}^{3}}\left|(-\Delta)^{\frac{j}{2}} \psi_{k}(t, x)\right|^{2} d x+c_{c} \int_{\mathbb{R}^{3}} \frac{\rho_{\psi}(t, x)}{|x|} d x \\
& +\frac{1}{2} c_{p} \int_{\mathbb{R}^{3}} \int_{\mathbb{R}^{3}} \frac{\rho_{\psi}(t, x) \rho_{\psi}(t, y)}{|x-y|} d x d y-\frac{3}{4} c_{s} \int_{\mathbb{R}^{3}} \rho_{\psi}^{\frac{4}{3}}(t, x) d x=\mathcal{E}_{J}(\phi) .
\end{aligned}
$$


Proof The proof is standard. The higher-order operator and the Slater terms do not bring any new difficulties, and we refer the reader to [18] for an explicit proof in the case of Hartree and Hartree-Fock, and to [15, 24] for a proof in a more general setting (see also [25] for the pseudo-relativistic case, that is, when the kinetic operator is given by $\sqrt{-\alpha^{2} \Delta_{x_{i}}+\alpha^{-4}}$ where $\alpha>0$ denotes Sommerfeld's fine structure constant).

To show that there exists a constant such that $\|\psi\|_{L_{T}^{\infty} H^{J}(\gamma)} \leq C$ for all $T>0$, we need to control the kinetic part of the energy which obviously depends on the parity of $J$. For this purpose, we need the following Gagliardo-Nirenberg inequality:

$$
\left\|(-\Delta)^{\frac{s}{2}} \psi\right\|_{L^{2}(\gamma)} \leq \eta\left\|(-\Delta)^{\frac{J}{2}} \psi\right\|_{L^{2}(\gamma)}^{\frac{s}{J}}\|\psi\|_{L^{2}(\gamma)}^{1-\frac{s}{J}} .
$$

Therefore, it is enough to focus on the leading term in the kinetic energy part, namely $(-1)^{J+1} \frac{\alpha\left(J \hbar^{2 J}\right.}{m^{2 J-1} c^{2 J-2}}\left\|\Delta^{\frac{J}{2}} \psi\right\|_{L^{2}(\gamma)}$. Let us assume that $J$ is odd. Then, using (14) with the mass conservation and the estimate

$$
\int_{\mathbb{R}^{3}} \rho_{\psi}^{\frac{4}{3}}(t, x) d x \leq \kappa\|\psi\|_{H^{\frac{3}{8}}(\gamma)}^{\frac{8}{3}},
$$

we infer that the leading term in the kinetic part dominates the Slater correction since $J>\frac{3}{8}$. Thus $\psi \in L_{T}^{\infty} H^{J}(\gamma)$. Now, assume $J$ is even, then again using (14) with the mass conservation and the estimates

$$
\begin{aligned}
& \int_{\mathbb{R}^{3}} \frac{\rho_{\psi}(x)}{|x|} d x \leq\|\psi\|_{L^{2}(\gamma)}^{2}+\|\psi\|_{L^{2}(\gamma)}\|\psi\|_{L^{\frac{8}{3}}(\gamma)} \leq\|\psi\|_{L^{2}(\gamma)}^{2}+\|\psi\|_{H^{\frac{3}{8}}(\gamma)^{\prime}} \\
& \int_{\mathbb{R}^{3}} \int_{\mathbb{R}^{3}} \frac{\rho_{\psi}(t, x) \rho_{\psi}(t, y)}{|x-y|} d x d y \leq \kappa\|\psi\|_{L^{2}(\gamma)}^{\frac{4}{3}}\|\psi\|_{L^{\frac{8}{3}}(\gamma)}^{\frac{8}{3}} \leq \kappa\|\psi\|_{L^{2}(\gamma)}^{\frac{4}{3}}\|\psi\|_{H^{\frac{3}{8}}(\gamma)}^{\frac{8}{3}},
\end{aligned}
$$

we infer that the leading term of the kinetic part dominates the potential part. Therefore $\psi \in L_{T}^{\infty} H^{J}(\gamma)$.

\section{Acknowledgements}

The author thanks the anonymous referees for their comments and careful reading of the manuscript.

\section{Funding}

The author would like to acknowledge the support provided by the Deanship of Scientific Research at King Fahd University of Petroleum \& Minerals for funding this work through project No. SR171003.

\section{Abbreviations}

SPS, Schrödinger-Poisson-Slater.

\section{Availability of data and materials}

Not applicable.

\section{Competing interests}

The author declares that he has no competing interests.

Authors' contributions

The author produced, read and approved the final manuscript.

\section{Publisher's Note}

Springer Nature remains neutral with regard to jurisdictional claims in published maps and institutional affiliations. 


\section{References}

1. Slater, J.C.: A simplification of the Hartree-Fock method. Phys. Rev. 81(3), 385 (1951)

2. Dirac, P.A.: Note on exchange phenomena in the Thomas atom. In: Mathematical Proceedings of the Cambridge Philosophical Society, vol. 26, pp. 376-385. Cambridge University Press, Cambridge (1930)

3. Bach, V.: Accuracy of mean field approximations for atoms and molecules. Commun. Math. Phys. 155(2), 295-310 (1993)

4. Bokanowski, O., Grebert, B., Mauser, N.J.: Local density approximations for the energy of a periodic Coulomb model. Math. Models Methods Appl. Sci. 13(08), 1185-1217 (2003)

5. Friesecke, G.: Pair correlations and exchange phenomena in the free electron gas. Commun. Math. Phys. 184(1), 143-171 (1997)

6. Carles, R., Lucha, W., Moulay, E.: Higher-order Schrödinger and Hartree-Fock equations. J. Math. Phys. 56(12), 122301 (2015)

7. Carles, R., Moulay, E.: Higher-order Schrödinger equations. J. Phys. A, Math. Theor. 45(39), 395304 (2012)

8. Kim, J., Arnold, A., Yao, X.: Global estimates of fundamental solutions for higher-order Schrödinger equations. Monatshefte Math. 168(2), 253-266 (2012)

9. Cycon, H.L., Foese, R.G., Kirsch, W., Simon, B.: Schrödinger Operators with Application to Quantum Mechanics and Global Geometry, study edn. Texts and Monographs in Physic, vol. 10. Springer, Berlin (1987)

10. Gorban, A.N., Karlin, I.V.: Schrödinger operator in an overfull set. Europhys. Lett. 42(2), 113-118 (2007)

11. Helffer, B.: Semi-Classical Analysis for the Schrödinger Operators Operator and Applications. Lecture Notes in Mathematics, vol. 1336of. Springer, Berlin (1988)

12. Karpeshina, Y., Shterenberg, R.: Extended states for polyharmonic operators with quasiperiodic potentials in dimension two. J. Math. Phys. 53, 103512 (2012)

13. Karpman, V., Shagalov, A.: Stability of solitons described by nonlinear Schrödinger-type equations with higher-order dispersion. Phys. D: Nonlinear Phenom. 144(1-2), 194-210 (2000)

14. Lucha, W., Rupprecht, H., Schöberl, F.F.: Significance of relativistic wave equations for bound states. Phys. Rev. D 46(3), 1088 (1992)

15. Bardos, C., Catto, I., Mauser, N., Trabelsi, S.: Setting and analysis of the multi-configuration time-dependent Hartree-Fock equations. Arch. Ration. Mech. Anal. 198(1), 273-330 (2010)

16. Zagatti, S.: The Cauchy problem for Hartree-Fock time-dependent equations. Ann. Inst. Henri Poincaré A 56, 357-374 (1992)

17. Castella, F.: L2 solutions to the Schrödinger-Poisson system: existence, uniqueness, time behaviour, and smoothing effects. Math. Models Methods Appl. Sci. 7(08), 1051-1083 (1997)

18. Chadam, J., Glassey, R.: Global existence of solutions to the Cauchy problem for time-dependent Hartree equations. J. Math. Phys. 16(5), 1122-1130 (1975)

19. Cancès, E., Le Bris, C.: On the time-dependent Hartree-Fock equations coupled with a classical nuclear dynamics. Math. Models Methods Appl. Sci. 9(07), 963-990 (1999)

20. Bokanowski, O., López, J.L., Soler, J.: On an exchange interaction model for quantum transport: the Schrödinger-Poisson-Slater system. Math. Models Methods Appl. Sci. 13(10), 1397-1412 (2003)

21. Keel, M., Tao, T.: Endpoint Strichartz estimates. Am. J. Math. 120(5), 955-980 (1998)

22. Kato, T.: On nonlinear Schrödinger equations. Ann. Inst. Henri Poincaré. Phys. Théor. 46(1), 113-129 (1987)

23. Cazenave, T.: Semilinear Schrödinger Equations, vol. 10. Am. Math. Soc., Providence (2003)

24. Trabelsi, S.: Global well-posedness for the mctdhf equations. Int. J. Math. Comput. 22(1), 134-143 (2013)

25. Hajaiej, H., Markowich, P.A., Trabelsi, S.: Multiconfiguration Hartree-Fock theory for pseudorelativistic systems: the time-dependent case. Math. Models Methods Appl. Sci. 24(3), 599-626 (2014)

\section{Submit your manuscript to a SpringerOpen ${ }^{\circ}$ journal and benefit from:}

- Convenient online submission

- Rigorous peer review

- Open access: articles freely available online

- High visibility within the field

- Retaining the copyright to your article

Submit your next manuscript at $>$ springeropen.com 